
allemande

51-1 | 2019

La République démocratique allemande dans l'espace public européen (1949-2018)

\title{
"Nous, les Allemands, nous sommes un peuple de réfugiés ». La mémoire des migrations dans la presse allemande durant l'été 2015
}

\section{Valérie Robert}

\section{(2) OpenEdition}

\section{Journals}

Édition électronique

URL : https://journals.openedition.org/allemagne/1622

DOI : 10.4000/allemagne. 1622

ISSN : 2605-7913

Éditeur

Société d'études allemandes

Édition imprimée

Date de publication : 2 juillet 2019

Pagination : 211-226

ISSN : 0035-0974

Référence électronique

Valérie Robert, « « Nous, les Allemands, nous sommes un peuple de réfugiés ». La mémoire des migrations dans la presse allemande durant l'été 2015 ", Revue d'Allemagne et des pays de langue allemande [En ligne], 51-1 | 2019, mis en ligne le 02 juillet 2020, consulté le 02 juin 2022. URL : http:// journals.openedition.org/allemagne/1622 ; DOI : https://doi.org/10.4000/allemagne.1622 


\section{«Nous, les Allemands, nous sommes un peuple de réfugiés "*. La mémoire des migrations dans la presse allemande durant l'été 2015}

\section{- Valérie Robert**}

L'été 2015 a vu l'Allemagne accueillir des centaines de milliers de réfugiés et se métamorphoser dans les médias allemands et internationaux en modèle d'une «culture de l'accueil» (Willkommenskultur), notamment grâce à la phrase d'Angela Merkel lors de sa conférence de presse du 31 août: «Wir schaffen das» («Nous allons y arriver»). L'impulsion pour l'accueil de ces réfugiés semblait ainsi venir du gouvernement fédéral. Pourtant, avant cette date, «Wir schaffen das» était devenu un topos dans la presse allemande, qui avait construit l'image d'une Allemagne se devant d'être accueillante parce qu'elle était elle-même le produit de migrations antérieures, dont la mémoire était pour cela réactivée.

\section{Journalisme, mémoire, identité}

Pour Barbie Zelizer, il faut considérer le journalisme «sous l'angle de la mémoire collective plus que de l'histoire ${ }^{(1)}$ : le journalisme médiatise la mémoire en un va-etvient entre présent et passé, le récit du passé étant une construction «fonctionnelle», «socio-politique» ${ }^{(2)}$, produit d'un processus de sélection et d'organisation, puisque

* Anne HÄHNIG, Julius LUKAS, Martin MACHOWECZ, «Weil wir wissen, wie es war», Zeit im Osten, 10 septembre 2015 (cahier pour les nouveaux Länder de Die Zeit, hebdomadaire suprarégional, siège: Hambourg, 2015/III: 496 946).

** Maître de conférences à I'Université Sorbonne Nouvelle-Paris 3, CEREG (Centre d'études et de recherches sur l'espace germanophone). Nous tenons à remercier très chaleureusement Ségolène Plyer pour sa relecture très attentive et ses remarques constructives.

1 Barbie Zelizer, «Journalism’s Memory Work», in: Astrid Erll, Ansgar Nünning (dir.), Cultural Memory Studies: An International and Interdisciplinary Handbook, Berlin, de Gruyter, 2008, p. 381.

2 Motti Neiger, Eyal Zandberg, Oren Meyers, "Reversed Memory: Commemorating the Past through Coverage of the Present », in: Barbie Zelizer, Keren Tenenboim-Weinblatt (dir.), Journalism and memory, Basingstoke, Palgrave Macmillan, 2014, p. 115. 
les journalistes ont le pouvoir de décider de qui va témoigner, et sous quel angle. Ils «fournissent une arène publique à différents acteurs» avec lesquels ils sont aussi en concurrence car ils «aspirent aussi à fournir leur propre lecture du passé collectif» ${ }^{(3)}$. On peut considérer les journalistes comme un «méta-agent» de la mémoire ${ }^{(4)}$, luimême pris dans un réseau de contraintes et d'intérêts spécifiques.

Les médias construisent des versions de la réalité et des versions du passé, qui «portent moins sur le passé » que sur des besoins présents ${ }^{(5)}$. On peut nommer ce rôle des médias, comme une variante de l'agenda-setting, le «collective-memory setting ${ }^{(6)}$ :

«[les] journalistes deviennent gatekeepers du passé comme ils le sont pour les événements de l'actualité immédiate. Ils trient, en lieu et place des historiens, ce qui va constituer notre histoire commune, et inscrivent ainsi certains sujets et moments dans la mémoire collective. [...] Les journaux permettent une sédimentation d'une mémoire collective qui se construit dans le partage d'événements vécus en commun, discutés, ressassés » ${ }^{(7)}$.

Et en effet, alors que l'arrivée des réfugiés est de manière répétée désignée comme un événement «historique», le discours et la comparaison avec les migrations antérieures ne sont pas le fait des historiens, même s'il y a eu avant la période considérée un débat à ce sujet entre historiens, mené aussi dans la presse ${ }^{(8)}$.

\section{Méthodologie}

Il s'agit ici de se pencher sur les modes de narration du passé dans une production journalistique historiquement et culturellement située. Les Cultural Studies considèrent les cultures comme des «communautés narratives» qui «se distinguent les unes des autres précisément en ce qui concerne leur réservoir narratif ${ }^{(9)}$, par la manière dont elles (se) racontent, notamment lorsque le récit de l'actualité réactive et ce faisant remodèle des récits antérieurs. Il s'agit alors d'identifier les «cadres narratifs préférentiels ${ }^{(10)}$, caractéristiques de chaque culture, le discours actualisant un stock de récits et de mémoires spécifiques ${ }^{(11)}$. Cela mène à se demander: pourquoi et

3 Motti Neiger, Oren Meyers, Eyal Zandberg, "On Media Memory: Editors' Introduction», in: ID. (dir.), On Media Memory. Collective Memory in a New Media Age, Basingstoke/New York, Palgrave MacMillan, 2011, p. 10.

4 Ibid., p. 11.

5 Astrid ErLL, «Medium des kollektiven Gedächtnisses: Ein (erinnerungs-)kulturwissenschaftlicher Kompaktbegriff», in: Astrid Erll, Ansgar NüNNING (dir.), Medien des kollektiven Gedächtnisses: Konstruktivität - Historizität - Kulturspezifität, Berlin/New York, de Gruyter, 2004, p. 4.

6 Ulrike KLEIN citée par Maren RöGER, «News Media and Historical Remembrance: Reporting on the Expulsion of Germans in Polish and German Magazine», in: Astrid Erll, Ann Rigney (dir.), Mediation, Remediation, and the Dynamics of Cultural Memory, Berlin/New York, de Gruyter, 2009, p. 189.

7 Marc Lits, Du récit au récit médiatique, Bruxelles, De Boeck, 2008, p. 135.

8 Patrice G. Poutrus, "Aus der Geschichte lernen? Zum Erkenntniswert des historischen Beispiels Flucht und Vertreibung der Deutschen aus Mittel- und Osteuropa», Neue Praxis, Sonderheft 13 (2016), p. 50.

9 Wolfgang Müller-Funk, Die Kultur und ihre Narrative. Eine Einführung, Vienne/New York, Springer, 2002, p. 15.

10 Erik Neveu, Sociologie du journalisme, Paris, La Découverte, 2009 (3éd.), p. 72.

11 Valérie Roвert, «Serialisierte Erzählung und kulturspezifische Pressenarrative. Die "Wulff-Affäre" in der deutschen Presse», DIEGESIS, Interdisziplinäres E-Journal zur Erzählforschung, 2 (2013): Erzählen im Journalismus, p. 51. 
comment ces récits sont-ils actualisés à ce moment précis et d'une certaine manière? Quelle est alors leur fonction? Les moments de «crise» agissent «comme catalyseurs de la production de valeurs et normes sociales au sens large ${ }^{(12)}$, cristallisent et font affleurer à la surface du discours, de manière très concentrée et souvent sous la forme de récits, des valeurs implicites le reste du temps ${ }^{(13)}$. Nous avons montré ailleurs que ceci vaut pour l'Allemagne dans les cas où la mémoire collective activée par les journalistes concerne leur identité en tant que groupe social ${ }^{(14)}$. Qu'en est-il lorsqu'il s'agit de l'identité du pays tout entier?

Pour répondre à ces questions, nous avons mené une étude interdisciplinaire sur la presse allemande à l'été 2015 , avec les outils de l'analyse du discours et de la narratologie. On a considéré l'ensemble des textes du corpus (présenté ci-dessous) comme un discours, un phénomène collectif dont les éléments sont reliés entre eux par un «système aux mailles serrées, dans lequel tout le monde s'oriente - plus ou moins en fonction des autres " ${ }^{(15)}$. Il s'agit de dégager la grammaire générale de ce discours, en examinant la représentation de migrations antérieures, leur re-médiatisation et la manière dont celle-ci en (re)construit la mémoire.

Le discours de presse se concentre sur le déroulement au jour le jour d'événements actuels, ici ce qui a été souvent désigné comme une "crise migratoire»; ceci constitue dans son ensemble un macro-texte qui peut être considéré comme une mise en récit de l'actualité(16), même si ses éléments pris individuellement ne sont pas nécessairement narratifs. Or le passé y trouve aussi sa place, comme à l'été 2015, où la mémoire de migrations précédentes constitue une sorte de ligne de basse continue, un des fils présents en permanence dans le tissu de l'information, plus ou moins mis au premier plan selon les cas et qui fournit une grille de lecture de l'actualité et oriente le macrorécit vers un horizon, celui de l'accueil. On distinguera donc ici ces deux niveaux: récit de l'actualité d'une part, dont l'issue n'est pas encore écrite, que l'on considérera comme le récit principal, et récit rétrospectif d'autre part, considéré comme un récit secondaire ${ }^{(17)}$ - c'est ce dernier qui nous intéresse ici, de même que la manière dont ces deux récits sont imbriqués.

\section{Corpus}

On a étudié la presse allemande entre le 10 août, qui marque le début de l'arrivée massive de réfugiés en Allemagne, et le 10 septembre 2015, cinq jours après

12 Jürgen Beule, Karl Otto Hondrich, «Skandale als Kristallisationspunkte politischen Streits», in: Ulrich SARCINELli (dir.), Demokratische Streitkultur: theoretische Grundpositionen und Handlungsalternativen in Politikfeldern, Bonn, Bundeszentrale für Politische Bildung, 1990, p. 144.

13 V. Robert, «Serialisierte Erzählung» (note 11), p. 55.

14 Ibid.

15 Hans Mathias Kepplinger, «Publizistische Konflikte. Begriffe, Ansätze, Ergebnisse», in: Friedhelm Neidhardt (dir.), Öffentlichkeit, öffentliche Meinung, soziale Bewegungen, Opladen, Springer, 1994, p. 223.

16 Marc Lits, «Le récit médiatique. Un oxymore programmatique ?», Recherches en communication, 7 (1997), p. 45.

17 Voir aussi Jocelyne Arquembourg, L'événement et les médias: les récits médiatiques des tsunamis et les débats publics, 1755-2004, Paris, Éditions des archives contemporaines, 2011, p. 43. 
l'ouverture des frontières par Angela Merkel. Le nombre d'articles sur les réfugiés explose mi-août ${ }^{(18)}$, lorsque le ministère fédéral de l'Intérieur annonce 800000 arrivées pour 2015. D’autres travaux ont montré l'importance de la mémoire pour l'ensemble de l'année $2015^{(19)}$; si nous nous concentrons ici sur cette courte période, c'est parce que, à l'été 2015, l'arrivée de réfugiés passant par la Hongrie et l'Autriche se télescope avec un phénomène mémoriel: les 70 ans de la fuite et de l'expulsion (Vertreibung), commémorés traditionnellement en septembre, après la journée de commémoration du 20 juin 2015 lors de laquelle le président fédéral Gauck avait déjà comparé Vertriebene (expulsés) et réfugiés ${ }^{(20)}$. On peut ajouter à cela ce qui n'est pas une commémoration mais une ressemblance évidente: l'arrivée de réfugiés se fait par la Hongrie et en août, comme celle de réfugiés de RDA en 1989.

Le corpus étudié est constitué d’articles publiés dans la presse (suprarégionale et régionale) quotidienne, magazine et en ligne. Si les titres de la presse suprarégionale sont les plus réputés, la presse régionale a une grande importance dans le paysage médiatique polycentrique allemand, certains titres ou groupes de titres ayant une audience souvent supérieure à celle des suprarégionaux ${ }^{(21)}$. Son audience cumulée était en 2015 de 49,7\% des plus de 14 ans, alors qu'elle n'était que de 4,2\% pour l'ensemble des quotidiens suprarégionaux «de qualité ». Les tabloïds (Bild à l'échelle nationale et 7 autres régionaux) avaient en 2015 une audience totale de $16,9 \%{ }^{(22)}$.

Pour constituer le corpus, on a d'abord listé les migrations susceptibles d'être évoquées ${ }^{(23)}$ puis utilisé la base de données Factiva (qui regroupe la plupart des quotidiens et magazines allemands) sans déterminer a priori les titres à considérer, ainsi que les archives de la Frankfurter Allgemeine (qui ne sont pas dans Factiva). On a pris en compte autant les textes d'information, les textes d'opinion que le courrier des lecteurs.

On a repéré et analysé, pour la période choisie, la co-occurrence de Flüchtling ${ }^{(24)}$ («réfugié», le terme le plus fréquemment utilisé( ${ }^{(25)}$ ) avec les items suivants et leurs variations - présentés ici par ordre d'importance numérique - qui font référence à des migrations passées:

18 Cf. Michael Haller, Die «Flüchtlingskrise» in den Medien. Tagesaktueller Journalismus zwischen Meinung und Information, Francfort-sur-le-Main, Otto Brenner Stiftung, 2017.

19 Katharina Eisch-Angus, «2015.1945. Erzählen von der Ankunft. Ein Versuch zum Flüchtlingsgedächtnis in der Willkommenskultur», in: Burkhard Pöttler, Katharina EIsCH-Angus, Johann Verhovsek (dir.), Fundstücke europäisch-ethnologischen Forschens. Eine Festschrift für Helmut Eberhart, Münster/New York, Waxmann, 2018, p. 225.

20 Stephan Scholz, «Willkommenskultur durch 'Schicksalsvergleich'. Die deutsche Vertreibungserinnerung in der Flüchtlingsdebatte», Aus Politik und Zeitgeschichte, 26-27 (2016), p. 41.

21 Valérie Robert, La presse en France et en Allemagne. Une comparaison des systèmes, Paris, Presses Sorbonne Nouvelle, 2011, p. 88.

22 Chiffres Media-Analyse de juillet 2015, www.agma-mmc.de/fileadmin/user_upload/Pressemitteilungen/2015/PM_ma_2015_Tageszeitungen.pdf.

23 On a utilisé pour cela Klaus J. BAde, Leo Lucassen, Pieter C. Emmer, Jochen Oltmer (dir.), Enzyklopädie Migration in Europa vom 17. Jahrhundert bis zur Gegenwart, Paderborn, Schöningh/Fink, 2007.

24 Travaillant sur la langue, nous avons laissé ici en allemand les termes étudiés.

25 Dans 91 \% des cas pour le corpus de Mike Berry, Inaki Garcia-Blanco, Kerry Moore et al., Press Coverage of the Refugee and Migrant Crisis in the EU: A Content Analysis of Five European Countries, Report prepared for the United Nations High Commission for Refugees, décembre 2015, www.unhcr. org/56bb369c9.pdf, p. 7. 
- Vertreibung (fuite et expulsion), Vertriebene (expulsés), Umsiedler ${ }^{(26)}$, Treck (convoi de réfugiés) : $38 \%$ des textes,

- fuite de RDA: DDR, 1989: $30 \%$,

- Gastarbeiter (travailleurs immigrés des années 1950 à 1970) : $14 \%$,

- mouvements migratoires postérieurs à 1945 venant d'autres pays du bloc de l'Est: Aussiedler (Allemands du bloc de l'Est), Russlanddeutsche (Allemands de Russie), Spätaussiedler («migrants tardifs»): $11 \%$,

- migrations ayant suscité un fort engagement humanitaire: Jugoslawien, 11 \%; Vietnam, 2,5 \%; Frühling et Prager (printemps et «de Prague»), 2,3 \%; Ungar (Hongr-) et 1956, Ungar et Aufstand (soulèvement), $2 \%$,

- Völkerwanderung (invasions barbares; cet item n'était pas prévu mais s'est imposé) : $9 \%$,

- période nazie: Exil, Jude (Juif), Evian, Flüchtlingskonferenz (conférence sur les réfugiés juifs) : $5 \%$,

- Hugenotten (Huguenots) : $3 \%$.

Une fois mis à part les articles sans analogie historique explicite, on a établi un corpus de plus de 400 textes, issus de 59 quotidiens (52 régionaux, 7 suprarégionaux) et de 11 magazines, hebdomadaires ou journaux du dimanche ${ }^{(27)}$. Dans la presse quotidienne, $24 \%$ des articles du corpus proviennent de la presse suprarégionale et $76 \%$ de la presse régionale.

\section{Qui parle?}

On sait que «la plupart des textes journalistiques mettent en scène des sources et intègrent du discours rapporté ${ }^{(28)}$ : dans notre corpus, les comparaisons historiques émanent à la fois des journalistes et des personnes auxquelles ils donnent la parole (politiques, citoyens, lecteurs dont on publie les lettres). Bien avant le 31 août, les analogies proviennent de nombreux députés au Bundestag ou des Länder, de ministres-présidents, mais aussi de nombreux maires, en première ligne face aux problèmes concrets d'organisation. On note également une forte présence de représentants des Églises; ainsi le chef de l'Église protestante de Rhénanie annonce dès le 22 août «Wir schaffen das», en se référant entre autres à son expérience personnelle de Vertriebener ${ }^{(29)}$.

26 Ce terme est celui qu'utilisait la RDA et dont on rencontre encore quelques occurrences (cf. Alice Volkwein, (Dis)cours mémoriel de la fuite et expulsion dans l'Allemagne unifiée (1989-2005). Complexe mémoriel et identitaire dans les sphères privée et publique, thèse pour le doctorat en études germaniques, Université Sorbonne Nouvelle-Paris 3, 2012, p. 6).

27 On indiquera lors de chaque citation la nature du média, son siège et sa diffusion payée au $3^{\mathrm{e}}$ trimestre 2015 (2015/III). Parfois, le chiffre de la diffusion est celui d'un groupe de titres qui partagent des contenus. Pour la presse régionale, meedia.de/2015/10/21/ivw-analyse-der-regionalzeitungen-keinegewinner-unter-den-80-top-titeln-dramatische-zahlen-in-berlin/; pour la presse quotidienne et magazine suprarégionale, meedia.de/2015/10/20/ivw-blitz-analyse-auflagen-verluste-der-magazinedramatischer-denn-je-nur-sieben-gewinner-in-der-top-50/ (pages consultées le 23.03.2018).

28 Roselyne Ringoot, Analyser le discours de presse, Paris, Armand Colin, 2014, p. 262.

29 Manfred Reкоwsкi, "Auf dem Schulhof war ich damals der Pole», Rheinische Post, 22 août 2015, quotidien régional, siège: Düsseldorf, 2015/III : 291260. 
Cependant, malgré «l'interaction avec d'autres discours sociaux ${ }^{\left({ }^{(0)}\right)}$, les producteurs du discours de presse sont bien les journalistes, qui sélectionnent la parole citée, produisent, suscitent, encadrent, mettent en scène ou renforcent les analogies historiques, dans un mouvement souvent circulaire. La comparaison semble d'ailleurs devenue un topos, qui encadre de façon quasi-systématique les propos rapportés.

L'activisme dans le collective-memory setting en faveur des réfugiés est très net dans notre corpus. Ainsi, la Neue Presse liste différentes vagues de réfugiés et en dit:

«Ils sont restés. Et sont aujourd'hui une partie utile de la société: professeurs, délégués du personnel, artistes et médecins, restaurateurs et entrepreneurs. À partir d'aujourd'hui, la $N P$ les présente dans une série. Des réfugiés qui ont saisi leur chance - et qui ne sont un problème pour personne» ${ }^{(31)}$.

Les récits de vie sont donc sollicités (ce qui découle entre autres des intérêts commerciaux de la presse) et insérés dans un cadre argumentatif spécifique.

\section{L'histoire comme leçon}

Le champ lexical du souvenir est très présent, comme performatif ( «nous nous souvenons » $\left.{ }^{(32)}\right)$, comme appel à se remémorer ce que parents ou grands-parents ont raconté, ou encore comme projet:

"Aujourd'hui, beaucoup de jeunes en ont à peine conscience: de nombreux Allemands ont également été, il y a des décennies, dans la situation d'arriver dans un pays étranger exactement comme c'est le cas des réfugiés de zones de guerre et de crise dans le monde qui arrivent aujourd'hui en Europe. La $N W Z$ veut rappeler ce fait. Elle appelle les gens qui ont eux-mêmes fait l'expérience de la fuite et de l'expulsion à se manifester. [...] La NWZ veut conserver et continuer à raconter ces histoires » ${ }^{(33)}$.

Il s'agit de réactiver la mémoire communicative, ou de constater cette réactivation: «Des souvenirs se réveillent» ${ }^{(34)}$.

L'analogie historique «tente explicitement de rendre le passé pertinent pour le présent en utilisant un événement passé comme outil pour analyser et prédire l'issue d'une situation actuelle ${ }^{(35)}$; le champ lexical de la leçon est très présent dans notre corpus. Il s'agit bien d'apprendre de l'histoire par l'activation d'une mémoire porteuse de normes et de valeurs, mobilisée en faveur de l'accueil des réfugiés : «Qui connaît le passé n’a pas le droit de rejeter les réfugiés. Qui voit le présent ne peut pas les rejeter. Qui pense à l'avenir ne les rejettera pas » ${ }^{(36)}$. La mémoire (dimension rétrospective du récit)

30 R. Ringoot, Analyser (note 28), p. 68.

31 «Kein Problem - eine Chance», Neue Presse, 15 août 2015, quotidien régional, siège: Hanovre, 2015/III incluse dans les chiffres de la Hannoversche Allgemeine Zeitung: 396589 exemplaires.

32 "Schwierige Entscheidung», Ostthüringer Zeitung, 2 septembre 2015, quotidien régional, siège: Gera (Thuringe), 2015/III: 252 577, incluant aussi la Thüringer Allgemeine et la Thüringische Landeszeitung.

33 Sandra Binkenstein, «Heimat in Friesland gefunden», Nordwest-Zeitung, ${ }^{\text {er }}$ septembre 2015, quotidien régional, siège: Oldenburg (Basse-Saxe), 2015/III : 199 392, incluant 7 quotidiens locaux.

34 ID., «Erinnerungen werden wach», Nordwest-Zeitung, 9 septembre 2015.

35 Jill A. EDY, «Journalistic Uses of Collective Memory», Journal of Communication, printemps 1999, p. 77.

36 Dieter Zetsche, patron de Daimler, in : « 100 STIMMEN GEGEN FLÜCHTLINGS-HASS », BILD am Sonntag, 30 août 2015, journal du dimanche suprarégional, siège: Berlin, 2015/III : 1082510. 
soutient la projection dans l'avenir (dimension prospective) et semble permettre un seul dénouement possible à la «crise», l'accueil des réfugiés (dimension prescriptive).

\section{Lieux de mémoire et généalogie des migrations}

Les textes établissent une généalogie des migrations que permet l'utilisation du terme Flüchtling, qui crée une cohésion entre les différentes migrations évoquées, comme entre les différents épisodes d'une série. Il met l'accent sur l'expérience de la fuite et de la souffrance, pour désigner à la fois les réfugiés de 2015, et - rétroactivement - des migrations précédentes. Prix Nobel de littérature, Hertha Müller, qui a fui la Roumanie en 1987, déclare ainsi: «Moi aussi, j’étais une réfugiée » ${ }^{(37)}$.

L'aspect quantitatif est très important dans notre corpus: les différentes migrations évoquées le sont de manière chiffrée, ce qui permet de comparer l'ampleur du phénomène et d'objectiver l'analogie. On constate aussi une addition des migrations, une généalogie qui devient un canon et que l'on retrouve sous forme de liste, plus ou moins complète, qui comprend toujours au moins les Vertriebene et, selon les cas, les réfugiés de RDA et (ou) les Gastarbeiter, qui en sont le noyau dur.

Au niveau local, on voit apparaître des lieux de mémoire, au sens propre: des lieux qui symbolisent cette histoire et dont la presse rappelle qu'ils ont vu passer toute une série de migrants. Il peut s'agir de villes, de villages, de bâtiments, de structures d'accueil, pour lesquels on évoque un mouvement cyclique ${ }^{(38)}$, ce qui renforce encore l'aspect sériel. Un lieu souvent évoqué est le camp de transit de Friedland: "À Friedland, on peut observer 70 ans d'histoire de la RFA comme avec une loupe ${ }^{(39)}$.

Cette addition, ce mille-feuilles de migrations est souvent incarné par des individus ou les différentes générations d'une famille, dans ce cas il s'agit de migrations interallemandes. Parmi plus d'une vingtaine de témoignages de personnes ayant fui la RDA, un professeur retraité raconte:

«Je me suis enfui trois fois dans ma vie. D’abord en 1945, de ce qui est aujourd'hui la Pologne, avec une charrette à bras en direction du Sud. De là nous avons été chassés vers l'Ouest en 1946, jusqu'en Thuringe. En 1957, je me suis enfui de RDA vers Berlin-Ouest. À mon avis : ce pays, qui a vécu une histoire de l'expulsion, doit proposer une patrie à des gens qui viennent d'ailleurs dans le monde et qui recherchent un asile ${ }^{(40)}$.

Ailleurs, un octogénaire explique qu'il s'occupe de réfugiés à la fois parce qu'il descend de protestants autrichiens ayant dû fuir au XVII siècle et parce qu'il a vécu la Vertreibung ${ }^{(41)}$.

37 «Ich war auch ein Flüchtling», BILD am Sonntag, 30 août 2015.

38 «Ehemalige Unterkunft wird wieder benötigt», Frankfurter Allgemeine, 2 septembre 2015, quotidien suprarégional, siège: Francfort-sur-le-Main, 2015/III : 231360.

39 «Für mehr als vier Millionen Menschen wurde Friedland das 'Tor zur Freiheit'», Hannoversche Allgemeine Zeitung, 26 août 2015, quotidien régional, siège: Hanovre, 2015/III : 396589 (ce chiffre inclut la diffusion de 17 autres quotidiens régionaux et locaux).

40 Anne Hähnig, Julius Lukas, Martin Machowecz, «Weil wir wissen, wie es war», Zeit im Osten, 10 septembre 2015 (cahier pour les nouveaux Länder de Die Zeit, hebdomadaire suprarégional, siège: Hambourg, 2015/III : 496 946).

41 Michael Kasperowitsch, «Fromm reden reicht ihm nicht», Nürnberger Nachrichten, 26 août 2015, quotidien régional, siège: Nuremberg, 2015/III : 248947. 


\section{La mémoire de la Vertreibung (expulsion des Allemands entre 1945 et 1949) et le retour des témoins individuels}

Parmi les migrations évoquées, la Vertreibung arrive en tête, ce qui tient d'abord à l'agenda commémoratif: le «Tag der Heimat» est fêté à partir du $1^{\text {er }}$ dimanche de septembre à Berlin puis ailleurs dans les semaines qui suivent ${ }^{(42)}$. Mais, en 2015, l'actualité donne à la mémoire de la Vertreibung une vigueur et une orientation nouvelles, qui dépassent largement l'acteur habituel qu'est le Bund der Vertriebenen (BdV, la fédération des Vertriebene, qui regroupe toutes les associations locales d'expulsés).

La Vertreibung est présentée comme la première migration et le premier accueil de masse de l'histoire de l'Allemagne après 1945, la migration originelle, la matrice des épisodes suivants. De plus, l'imagerie de la fuite est, dans le discours mémoriel allemand, avant tout celle des Vertriebene. L'emploi de Treck (le terme désignant un convoi de réfugiés) renvoie automatiquement à cette période, et il devient un mot-clé qui opère la connexion entre actualité et mémoire.

Le récit n'évoque à peu près jamais les causes de l'expulsion et les crimes nazis, mettant l'accent sur le statut de victimes des Vertriebene, qui permet l'analogie: le présent déteint donc sur la représentation du passé. Il est certes rappelé qu'il s'agissait d'une migration «interne» d'Allemands, mais ce qui est davantage souligné, ce sont certes les discriminations dont les Vertriebene ont fait l'objet, mais aussi le fait que leur intégration à long terme peut être considérée comme un succès. Ces différents aspects sont présents dans les témoignages sélectionnés et publiés par la presse, le plus souvent présentés comme des arguments en faveur d'un accueil chaleureux des réfugiés. Ce faisant, on fait disparaître l'éventuelle "ambivalence» dans les positions de Vertriebene ou de leurs descendants face à l'analogie ${ }^{(43)}$.

Le lien avec la Vertreibung s'effectue donc particulièrement par la représentation de la souffrance passée, condition de l'empathie, un aspect devenu plus important depuis les années 2000 avec le développement d'une vision des Vertriebene comme victimes, qui a rendu possible de «comparer les destins " ${ }^{(44)}$. À ceci s'ajoute le fait que la mémoire de la Vertreibung, après avoir été instrumentalisée politiquement ${ }^{(45)}$, ce qui faisait passer les expériences individuelles à l'arrière-plan ${ }^{(46)}$, a après 1990 perdu son caractère politique pour s'inscrire dans un «discours universaliste des droits de l'homme». Cette évolution a aussi permis d'ancrer la Vertreibung dans l'histoire des migrations ${ }^{(47)}$.

Même si les témoignages et récits individuels sont, dans l'ensemble du corpus, majoritaires, l'acteur institutionnel qu'est le Bund der Vertriebenen reste présent, qui passe progressivement d'un refus de la comparaison à un appel à l'empathie et à l'aide

42 Stephan Scholz, "Gedenktage», in: Stephan Scholz, Maren Röger (dir.), Die Erinnerung an Flucht und Vertreibung. Ein Handbuch der Medien und Praktiken, Paderborn, Schöningh, 2015, p. 175.

43 K. Eisch-Angus, «2015.1945» (note 19), p. 230.

44 S. ScholZ, «Willkommenskultur» (note 20), p. 45.

45 Rainer Münz et Rainer OHliger, «Auslandsdeutsche», in : Étienne François, Hagen Schulze (dir.), Deutsche Erinnerungsorte, Bd. I, Munich, Beck, 2001, p. 385.

46 Arnd BAUERKÄMPER, «Deutsche Flüchtlinge und Vertriebene aus Ost-, Ostmittel- und Südosteuropa in Deutschland und Österreich seit dem Ende des Zweiten Weltkriegs", in: K. J. BADE et al., Enzyklopädie Migration (note 23), p. 484.

47 S. Scholz, «Willkommenskultur» (note 20), p. 45. 
pour les demandeurs d'asile, même si son président B. Fabritius insiste sur le fait que les réfugiés "viennent de cultures très différentes, avec des valeurs et des visions qui en partie ne sont pas compatibles avec notre consensus de base ${ }^{(48)}$. Or, ce discours institutionnel est concurrencé et souvent contredit par les témoignages individuels recueillis par la presse: on assiste à un retour des "expulsés 'anonymes'» ${ }^{(49)}$ dans le discours mémoriel, en particulier au niveau local. Cependant, la comparaison semble davantage renforcer la mémoire de la Vertreibung que permettre réellement d'appréhender le vécu des réfugiés contemporains, auxquels on donne très peu la parole. La nette individualisation du discours ne concerne que la mémoire des migrations antérieures, pas l'actualité directe ${ }^{(50)}$.

La présence des Vertriebene dans la presse n'est pas de même nature selon l'échelle, locale, régionale ou suprarégionale. La presse régionale, souvent dans ses pages locales, consacre de très nombreux textes aux Vertriebene, ce qui s'explique par un fort maillage commémoratif local ${ }^{(51)}$ : monuments, associations (Landsmannschaften), événements (expositions, messes, pèlerinages...). Fortement ancrée dans sa région, cette presse y a de nombreux lecteurs fidèles, majoritairement de plus de 40 ans ${ }^{(52)}$ donc susceptibles d'avoir été touchés directement ou indirectement par la Vertreibung. Elle transpose à l'échelle locale et micro-locale les thématiques générales, visant à susciter chez les lecteurs un sentiment de familiarité ou d'identification ${ }^{(53)}$. La forte présence au niveau local de la mémoire individuelle est aussi la conséquence d'une stratégie commerciale, qui mise sur le sentiment de proximité. Le niveau local va donc de pair avec un accent mis sur l'individu, et il semble que la conjonction de la commémoration et de l'actualité favorise la forte présence d'une mémoire individuelle ${ }^{(54)}$, d'une «mémoire communicative» qui semble provisoirement prendre le pas sur la «mémoire culturelle» - ce qui est beaucoup moins le cas dans la presse suprarégionale. Ainsi, un habitant explique que c'est le contexte et la médiatisation du nouveau phénomène qui l'ont poussé à retrouver le récit écrit par sa grand-mère et à interroger son père ${ }^{(55)}$. La mémoire de la Vertreibung est bien «en perpétuel changement » ${ }^{(56)}$.

Dans le contexte de l'été 2015, nombreux sont les Vertriebene auxquels est donnée la possibilité d'exprimer leurs souvenirs individuels. Dans la plupart des cas, ces récits sont encadrés par une analogie et une leçon, ils ne se suffisent donc pas à eux-mêmes. La proportion entre récit de vie et leçon à en tirer est variable: dans la presse locale, c'est le témoignage qui prend le plus de place, alors que dans la presse suprarégionale,

48 «Das macht den Menschen Angst // Vertriebenen-Präsident Bernd Fabritius will Wirtschaftsflüchtlinge schneller zurückschicken - und fordert gleichzeitig mehr Solidarität mit den Opfern von Vertreibung», Der Tagesspiegel, 22 août 2015, quotidien régional, siège: Berlin, 2015/III: 95737.

49 A. VolKwein, «(Dis)cours mémoriel» (note 26), p. 483.

50 M. Haller, Die «Flüchtlingskrise» (note 18), p. 133.

51 Münz/Ohliger, «Auslandsdeutsche» (note 45), p. 381.

52 V. Robert, La presse (note 21), p. 88.

53 Claas Neumann, «Lokalpresse», in: Scholz/Röger (dir.), Die Erinnerung (note 42), p. 266.

54 Comme en témoigne K. Eisch-Angus, «2015.1945» (note 19).

55 Jan Melzer, «Flüchtlinge, meine neuen Nachbarn», Hamburger Abendblatt, 22 août 2015, quotidien régional, siège: Hambourg, 2015/III : 170977.

56 M. Röger, «News Media» (note 6), p. 199. 
il est condensé à l'extrême. Il y a bien une identité spécifique de la presse locale; les quotidiens suprarégionaux, quant à eux, ont davantage l'ambition de peser sur le débat public, d'être cités et repris par les autres médias ${ }^{(57)}$. Il faut donc analyser les médias dans leur (éco)système ${ }^{(58)}$. L'audience, et par là la situation financière des médias sont des aspects très importants, aussi pour le discours mémoriel: "C’est bien parce que le public a, au tournant des années 2000, répondu présent que les médias ont découvert la fuite et expulsion comme 'produit'» ${ }^{(59)}$.

\section{La fuite de RDA}

La mémoire de la fuite de RDA, elle aussi une migration interallemande, est aussi très présente, tout particulièrement celle de l'été 1989. La référence passe dans ce cas largement par les symboles: rideau de fer vs. barbelés installés par la Hongrie en août 2015, ou encore les trains - ceux ayant amené les réfugiés de RDA de Hongrie en Autriche puis en RFA, et ceux amenant les réfugiés de 2015 d'Autriche en Allemagne. Les images de 2015 sont inscrites dans une série: "Les images se ressemblent» ${ }^{(60)}$. Contrairement à celle de la Vertreibung, la mémoire est ici moins celle de l'événement lui-même que celle de sa médiatisation sur le moment.

Les récits de fuite de RDA dans lesquels on ne rencontre pas d'analogie historique semblent faire partie du répertoire habituel dans la presse locale des nouveaux Länder, mais on rencontre dans notre corpus beaucoup de comparaisons à visée argumentative:

"Ce que les Syriens et les Africains font aujourd'hui, c'est ce que les Allemands de l'Est ont également fait en 1989 et 1990 . Ça vaut toujours le coup de s'enfuir, si on ne peut plus vivre quelque part. C'est quelque chose qu'il n'est pas difficile de comprendre. Même un nazi est capable de le comprendre ${ }^{(61)}$

déclare un ancien dissident de RDA dont un journal fait le portrait.

Ce message est donc spécifiquement adressé aux habitants des nouveaux Länder et critique les actes et attitudes xénophobes plus répandus à l'Est, par exemple dans cet éditorial de la Leipziger Volkszeitung:

"À l'époque, il y a 26 ans, des centaines de milliers de citoyens de RDA étaient en fuite vers l'Ouest. Ils voulaient une vie meilleure. Dans l'ancienne RFA, personne n'a demandé: Es-tu vraiment persécuté par la Stasi? As-tu vraiment des motivations politiques? Est-ce que peut-être tu trouves simplement la RDA trop limitée? Est-ce que par hasard tu ne serais pas un migrant économique?» ${ }^{(62)}$

57 C’est par exemple le cas pour «100 STIMMEN GEGEN FLÜCHTLINGS-HASS» (note 36), qui sera largement cité par les autres journaux.

58 Astrid Erll, Ann Rigney, "Introduction: Cultural Memory and its Dynamics», in: ID., Mediation (note 6), p. 9.

59 A. Volkwein, «(Dis)cours mémoriel» (note 26), p. 497.

60 «Die Bilder gleichen sich», Mitteldeutsche Zeitung, 2 septembre 2015, quotidien régional, siège: Halle (Saxe-Anhalt), 2015/III : 186669.

61 Zoran PAntic, "Andora hat genervt - und durfte die DDR doch noch verlassen », Neue Presse, 18 août 2015.

62 Jan Emendörfer, «Es sind genug Bananen für alle da», Leipziger Volkszeitung, 27 août 2015, quotidien régional, siège: Leipzig, 2015/III : 174038. 
Autre exemple d'analogie visant uniquement le public de l'Est: la Zeit publie le 10 septembre des appels de personnes ayant fui la RDA dans le cahier «Est », destiné uniquement aux nouveaux Länder.

\section{Réfugiés étrangers et démarche humanitaire}

On trouve également, dans une moindre mesure, des références à des migrations qui ne sont pas inter-allemandes mais qui réunissent à la fois l'imagerie de la fuite et la réalité de l'accueil. Les réfugiés d'ex-Yougoslavie notamment sont souvent cités, ce qui réactive une analogie rétrospective: les déplacements de populations dans les années 1990 ont en effet contribué "à réveiller les souvenirs de l'expulsion des Allemands» (63) et à faire naître une représentation des Vertriebene comme ayant subi un «nettoyage ethnique ${ }^{(64)}$. Mais la comparaison est surtout numérique, le récit de vie individuel n’a que rarement lieu, il s'agit alors de personnalités ou de lettres de lecteurs. Par ailleurs, la presse distingue entre les réfugiés des guerres des années 1990, dont l'accueil doit servir d'exemple, et les demandeurs d'asile actuels venus des Balkans, que l'on désigne comme des Wirtschaftsflüchtlinge («migrants économiques»), s'inscrivant ainsi dans la discussion politique sur l'accueil à réserver aux migrants ne venant pas de pays en guerre.

Les réfugiés hongrois de 1956 et tchèques de 1968 sont très peu évoqués, alors même qu'à l'époque, presse et opinion publique s'étaient largement mobilisées en leur faveur, en s'appuyant déjà sur l'analogie historique avec la Vertreibung ${ }^{(65)}$. En 2015, l'évocation de ces migrations a surtout pour fonction d'appeler les gouvernements de ces deux pays à accueillir des réfugiés, comme l'Allemagne l'avait fait à l'époque ${ }^{(66)}$, une expérience censée leur servir au sens propre de leçon. La référence aux boat-people vietnamiens de la fin des années 1970 est encore plus rare, alors que leur accueil a suivi une très forte médiatisation menant à une mobilisation tant de la presse que de la population ${ }^{(67)}$, entraînant d'ailleurs la création de la catégorie de "réfugié humanitaire». Cette césure dans l'histoire de l'Allemagne comme pays d'asile est pourtant largement occultée, ce qui pourrait s'expliquer par l'imagerie différente (sauvetage en mer $v s$. colonnes de réfugiés), ainsi que par un parcours géographique très différent.

\section{Les migrations d'avant 1945 : un vaste panorama}

Pour justifier l'accueil des réfugiés, on évoque souvent le fait que de nombreux Allemands ont dû fuir l'Allemagne pour des raisons politiques ou de simple survie

63 A. Volkwein, «(Dis)cours mémoriel» (note 26), p. 263.

64 S. Scholz, «Willkommenskultur» (note 20), p. 44.

65 Cf. Patrice G. Poutrus, «Refugee Reports:Asylum and Mass Media in Divided Germany during the Cold War and Beyond», in: Cornelia Wilhelm (dir.), Migration, Memory and Diversity. Germany from 1945 to the Present, New-York/Oxford, Berghahn Books (Studies in Contemporary European History), 2017, p. 93 et 99.

66 Hans-Jörg Sснміdт, «Aus meiner Sicht: Die Tschechen wollen ihre Ruhe», Sächsische Zeitung, 22.08.2015.

67 Cf. Olaf Beuchling, «Vietnamesische Flüchtlinge in West-, Mittel- und Nordeuropa seit den 1970er Jahren», in: K.J. BAde et al., Enzyklopädie Migration (note 23), p. 1072; Julia KLeinschmidt, «Die Aufnahme der ersten 'boat people' in die Bundesrepublik», Deutschland Archiv Online, 26.11.2013, www.bpb.de/170611 (page consultée le 25.03.2019). 
matérielle. Différentes vagues sont alors évoquées: l'exil d'intellectuels comme Heine ou encore l'émigration vers les États-Unis au XIX ${ }^{\mathrm{e}}$ siècle. La ville de Passau, «la porte d'entrée principale vers l'Allemagne» en 2015, est désignée comme «l'Ellis Island de l'Allemagne " ${ }^{(68)}$. Un chapitre particulier est celui de l'exil pendant la période national-socialiste, qui est une référence bien moins fréquente que la Vertreibung, même si le passé nazi reste la toile de fond de tout le discours et affleure davantage après les attaques contre des foyers de réfugiés. Le rappel associe alors exil, persécutions et Shoah en un continuum, un contre-modèle absolu contre lequel il s'agit de se positionner - en même temps que contre les attitudes de rejet et les attaques envers des réfugiés en 2015, considérées comme une répétition. Ce contre-modèle impose d'accueillir les réfugiés actuels. La mémoire individuelle des survivants de la Shoah est alors mobilisée: «les réfugiés sont les juifs d'aujourd'hui» ${ }^{(69)}$.

La presse allemande remonte aussi beaucoup plus loin dans le temps: la référence principale est alors l'accueil des Huguenots et des Vaudois. Elle est particulièrement présente dans la presse locale, pour souligner le contraste entre des attaques contre des foyers de réfugiés et une tradition d'accueil présentée comme séculaire, et combine en général l'aspect humanitaire et l'intérêt économique d'accueillir des migrants qualifiés.

\section{Les Gastarbeiter et leur intégration ratée}

Les Gastarbeiter, travailleurs immigrés arrivés en Allemagne à partir du milieu des années 1950, sont également évoqués dans le discours sur les réfugiés. Leur statut est différent, mais c'est bien leur présence qui a transformé la RFA en un pays d'immigration de fait. La comparaison est souvent faite par les politiques et s'inscrit dans un discours sur les leçons à tirer, cette fois, des erreurs du passé. Autant les Vertriebene et les immigrés de RDA sont présentés comme des exemples d'intégration réussie, autant la politique vis-à-vis des travailleurs immigrés, longtemps considérés comme de passage, est vue comme un échec qu'il ne faut pas reproduire. Les Gastarbeiter sont considérés comme un groupe homogène dont la principale qualité reste d'avoir aidé à l'essor économique de l'Allemagne. S'ils sont mobilisés comme modèle positif, c'est sous cet angle, pour exhorter à accueillir des réfugiés censés pouvoir eux aussi remplir ce rôle. Ce discours émane particulièrement de chefs d'entreprise, notamment dans des déclarations du patron de Daimler reprises dans toute la presse.

C'est probablement parce que les Gastarbeiter ne sont pas considérés comme des réfugiés que, dans leur cas, les récits de vie sont très rares. Ils relèvent plus des statistiques que d'une référence individuelle et émotionnelle: seules des stars comme le footballeur Ilkay Gündogan ${ }^{(70)}$ ont l'occasion d'évoquer très brièvement l'expérience de leurs parents ou grands-parents. Cette migration semble ne pas disposer non plus d'images iconiques propres à susciter l'empathie, elle est presque entièrement subsumée sous le thème de l'intégration:

68 Walter Wüllenweber, «Der Ansturm», Stern, 27 août 2015, hebdomadaire suprarégional, siège: Hambourg, 2015/III : 412737.

69 Oliver PolAK, «Die Lösung», Welt am Sonntag, 30 août 2015.

70 «Nationalmannschaft; Gündogan: “Ohne Zuwanderer wäre Deutschland nie Weltmeister” ", Berliner Morgenpost Online et Hamburger Abendblatt, 2 septembre 2015. 
«Le véritable défi, c’est l'intégration. La bonne nouvelle: en ce qui concerne l’intégration, l'Allemagne a déjà fait dans le passé toutes les erreurs imaginables. Elle n'a pas besoin de les refaire ${ }^{(71)}$.

\section{Fonctions de la mémoire et du récit}

L'accueil est donc l'horizon du récit, qui pour cela s'appuie sur l'activation de la mémoire de migrations antérieures, porteuse d'un modèle de comportement, d'une ou de plusieurs morales liées au contexte. La généalogie des migrations établie à l'été 2015 est spécifique à ce moment ${ }^{(72)}$ parce qu'elle remplit alors la fonction d'une tradition positive dans laquelle s'inscrire, ce qui suppose cependant de gommer de nombreux aspects; la société de l'été 2015 semble avoir besoin d'un mythe fondateur, celui d'un pays-refuge. Pour cela, on re-raconte, on ré-écrit aussi le récit des épisodes de l'histoire qui vont dans ce sens et qui servent de leçon: c'est surtout parce que des Allemands ont été des réfugiés et que d'autres Allemands les ont accueillis qu'il faut, en 2015, accueillir des réfugiés étrangers. Il s’agit donc de construire une pré-histoire construite et transmise médiatiquement qui instaure une série autant qu'elle s'y inscrit. Ce récit secondaire accompagne le récit principal, la masse de textes plus factuels produits sur la «crise migratoire», et lui fournit un cadre interprétatif.

La mémoire a ici une fonction argumentative, qui la forme en retour. L'analogie historique peut d'ailleurs être considérée comme une manière d'exprimer une opinion tout en gardant une apparence de neutralité ${ }^{(73)}$. Cette «neutralité» est une exigence spécifique du discours de presse en Allemagne dans lequel, depuis 1945 et l'importation du modèle anglo-saxon, on distingue pour cela - au moins formellement - les textes à fonction d'information de ceux exprimant une opinion ${ }^{(74)}$. Or, on constate dans notre corpus que l'analogie historique a valeur d'argument tout autant dans des textes d'information que dans des éditoriaux. Sélection et mise en scène de la mémoire d'une part et opinion d'autre part vont de pair. Comme souvent en Allemagne, les journalistes ne sont pas seulement des observateurs et des médiateurs neutres, mais sont aussi, tout autant que les politiques voire en rivalité avec eux, acteurs à la fois du discours mémoriel et de la politique ${ }^{(75)}$ : à l'été 2015 , il s'agit de construire, non plus de l'altérité (alors que le discours public, particulièrement en Allemagne, a traditionnellement tendance à considérer les migrants comme des «autres " $\left.{ }^{(76)}\right)$, mais de l'identité (dans les deux sens du terme), de bâtir un consensus non d'exclusion mais d'inclusion par le biais de l'analogie historique et de la mobilisation de la mémoire. La presse allemande, largement favorable à l'accueil des réfugiés, développe l'image d'une Allemagne qui devrait tirer des leçons du passé et admettre qu'elle est bien un pays d'immigration - et ce non seulement d'un point de vue social et culturel, mais

71 W. Wüllenweber, «Der Ansturm» (note 68).

72 S. Scholz, «Willkommenskultur» (note 20), p. 42.

73 J.A. EDy, «Journalistic Uses» (note 35), p. 77.

74 V. RoBert, La presse (note 21), p. 148.

75 Valérie RoBERT, «Acteurs ou témoins? Le récit des affaires politiques dans la presse française et allemande», in: Vincent Goulet et Christoph VATter (dir.), Champs médiatiques et frontières dans la "Grande Région" SaarLorLux et en Europe, Sarrebruck, Presses universitaires de la Sarre, p. 262. 
aussi et surtout sur le plan des représentations. Dans le contexte de 2015, il s'agit bien de "construire l'histoire des migrations comme une partie de l'histoire nationale » ${ }^{(77)}$ et, plus largement, de construire l'identité de la RFA dans le présent et dans le futur et, pour cela, de développer une histoire positive spécifiquement allemande.

\section{Critiques de la généalogie des migrations: un contre-discours}

Ce recours orienté à la mémoire est aussi critiqué, le plus souvent dans le courrier des lecteurs, donc à la marge du discours dominant. Ces voix minoritaires, opposées à ou inquiètes face à l'accueil des réfugiés, mettent en doute la pertinence des analogies, particulièrement celle avec les Vertriebene et, dans les nouveaux Länder, avec la fuite de RDA. Elles développent à la place une contre-analogie, la référence aux invasions barbares, Völkerwanderung. En allemand, traditionnellement, ce terme évoque moins la fin d'une civilisation que le fait que des populations venues de l'Est aient été force de changement. Mais cette perspective évolue en 2015: l'Allemagne et l'Europe sont de plus en plus assimilées à l'Empire romain menacé par une invasion ${ }^{(78)}$. Ce passage d'une connotation positive à une connotation négative se produit en parallèle d'une naturalisation du terme qui, d'abord utilisé avec des guillemets, apparaît de plus en plus sans cette marque de distance, le plus souvent dans les propos de politiques, et avec pour fonction de servir de contre-argument. La presse conteste le plus souvent la pertinence de cette comparaison. Des quotidiens locaux assument alors le rôle d'une encyclopédie en rappelant les connaissances historiques sur la Völkerwanderung; la Ostthüringer Zeitung va jusqu'à reproduire un article de Wikipedia ${ }^{(79)}$. Le recours à un discours «savant» sert alors à déconstruire une représentation de l'histoire qui ne va pas dans le sens du discours dominant.

Face à ce dernier émerge également, venant notamment du mouvement Pegida mais aussi de médias "alternatifs», une critique virulente des médias "établis» et de la Lügenpresse (la presse qui ment), accusée tout particulièrement de ne pas dire la vérité sur les réfugiés ${ }^{(80)}$.

\section{La fin du «conte d'été»}

Michael Haller a pour sa part, à partir d'un corpus allant de février 2015 à mars 2016, constaté non pas que les informations données par la presse auraient été fausses, mais que les opinions divergentes n'étaient pas assez représentées (ou uniquement «de manière méprisante») pour que soit garanti le pluralisme de l'information ${ }^{(81)}$. Dans notre corpus, lorsque l'opposition à l'accueil des réfugiés est évoquée, c'est sous sa

77 Philipp Seuferling, "To arrive means being able to tell». Memory Cultures and Narratives of Historical Migration in German Media in 1991-1994 and 2015-2017, mémoire de master, Department of Communication and Media, Lund University, 2017, p. 53. Nous remercions l'auteur de nous avoir communiqué son mémoire avant la parution de l’article qu'il en a tiré, in: Tobias Linné (dir.), Excellent MSc Dissertations 2017, Media and Communication Studies, Lund University, Lund, Faculty of Social Sciences, 2018, p. 103-170. Cf. Mischa Meier, «Die 'Völkerwanderung'», Aus Politik und Zeitgeschichte, 26-27 (2016).

80 M. Haller, Die «Flüchtlingskrise» (note 18), p. 1.

81 Ibid., p. 134. 
forme la plus radicale, celle de manifestations violentes, comme à Heidenau à partir du 21 août 2015, ou de l'incendie de centres d'accueil. Ce pendant négatif à la Willkommenskultur est toujours présent en arrière-plan, avec sa mémoire et ses représentations qu'on pourrait nommer iconiques, sans qu'il soit besoin de remonter à la période nazie, par exemple les émeutes anti-étrangers du début des années 90 à Rostock. Mais cette opposition reste présentée comme une exception qui semble ne pas remettre en question l'identité de l'Allemagne comme pays d'accueil.

Or, à l'automne 2015, l'opinion publique semble évoluer dans un sens contraire. Dans des sondages, à l'automne 2015, une large majorité était favorable à l'accueil des réfugiés; mais en janvier 2016, deux tiers des personnes interrogées se sentaient menacées par l'arrivée des réfugiés, et en octobre 2015, seules $25 \%$ étaient d'avis que la presse avait donné une image correcte des réfugiés ${ }^{(82)}$. Critique de la politique d'accueil et critique des médias se rejoignent, ce à quoi la presse va réagir progressivement en donnant de plus en plus la parole aux opposants à l'accueil, jusqu'à basculer dans une représentation globalement négative des réfugiés après les événements du Nouvel An 2015-2016 à Cologne. À cette occasion sont réactivées d'autres mémoires, par exemple celle d'hommes non-blancs comme un danger pour les «femmes allemandes», notamment avec des Unes montrant des mains noires sur des corps de femmes blancs (Focus, Süddeutsche Zeitung), qui rappellent les représentations des soldats coloniaux durant l'occupation de la Ruhr.

Pour Haller, tout se passe comme si les journalistes compensaient a posteriori l'absence de voix discordantes dans la presse dans les mois qui ont précédé(83). Le discours empathique - vis-à-vis des réfugiés - et euphorique - vis-à-vis d'une nouvelle Allemagne censée se réinventer en pays d'accueil sur la base de la mémoire des migrations précédentes - s'est retourné. Au plus tard avec Cologne, l'heure n'est plus à l'analogie historique positive. Alors que l'exclusion de l'«autre», le non-européen, le non-chrétien, le non-blanc, avait été temporairement levée au nom d'une souffrance partagée, même à 60 ans d'écart, ce discours est suivi par un retour brutal, dans les représentations données par les médias, de «l'autre», tout particulièrement de l'homme étranger (alors que la question du genre n'avait pas été posée en été). On assiste donc, après le "conte d'été» (Sommermärchen, terme utilisé durant l'été 2015, en référence à la Coupe du monde de football en Allemagne à l'été 2006), à un revirement du discours, centré ensuite majoritairement sur les problèmes censément liés à l'arrivée de réfugiés. La parole qui avait été de fait exclue précédemment devient de plus en plus présente (et présentable), parallèlement et probablement en réponse à une critique virulente et de plus en plus bruyante des médias, en particulier quant au traitement de l'immigration. 


\section{Résumé}

Vertriebene (expulsés), réfugiés de RDA ou encore Gastarbeiter (travailleurs immigrés): telles sont les migrations auxquelles est comparé l'afflux de réfugiés à l'été 2015. À côté de l'information sur l'actualité, la presse allemande, en constituant une généalogie des migrations censées avoir fait l'Allemagne d'aujourd'hui, opère un "collective-memory setting». Elle réactive et re-modèle la mémoire dans un récit à fonction prescriptive, une "construction fonctionnelle " porteuse d'un modèle de comportement et dont l'horizon est l'accueil des réfugiés. La généalogie des migrations établie à l'été 2015 remplit alors la fonction d'une tradition positive dans laquelle s'inscrire, construit une identité, celle d'un pays-refuge, d'un pays dont la ligne directrice ne peut être que "wir schaffen das». À l'été 2015, les journalistes ne sont pas seulement des observateurs et des médiateurs neutres, mais sont aussi acteurs à la fois du discours mémoriel et de la politique. C'est ce qui leur sera reproché à l'automne, après la phase d'euphorie, lorsque ce discours mémoriel et sa fonction seront critiqués en même temps que les médias qui les ont relayés et produits.

\section{Zusammenfassung}

Im Sommer 2015 wird in der deutschen Presse, parallel zur aktuellen Berichterstattung über die Ankunft in Europa Tausender Flüchtlinge, auf als vergleichbar dargestellte historische Präzedenzfälle zurückgegriffen. Unter anderem Vertriebene, DDR-Flüchtlinge, Gastarbeiter bilden durch eine explizite Serialisierung eine Genealogie der Migrationen, welche Deutschland geprägt haben sollen. Dadurch findet in der Presse ein „collective-memory setting“ statt, es wird retrospektiv ein "funktional konstruiertes" Gedächtnis aktiviert, welches wiederum als Interpretationsmuster der Aktualität eine preskriptive und normative Funktion innehat: Horizont des medial vermittelten Gedächtnisses ist die Aufnahme der Flüchtlinge. Die Genealogie der Migrationen schafft eine positive Identität des heutigen Deutschland als Zufluchtsland, dessen Motto nichts anderes „wir schaffen das"sein kann. Die Journalisten werden dadurch zu Akteuren des öffentichen Lebens und der Politik. Im Herbst 2015 aber endet das „Sommermärchen“; es werden gleichzeitig die Aufnahme von Migranten und die Haltung der Presse diesem Thema gegenüber in Frage gestellt. 